\title{
Cerebrotendinous xanthomatosis: clinical and MRI study (a case report)
}

\author{
Marco Fiorelli, Vittorio Di Piero, Stefano Bastianello, Luigi Bozzao, Antonio Federico
}

\begin{abstract}
We report a patient with cerebrotendinous xanthomatosis (CTX) presenting with dementia, spastic tetraparesis and an unreported akinetic-rigid syndrome. Computed tomography (CT) showed only cerebellar abnormalities while magnetic resonance imaging (MRI) detected additional pallidal and mesencephalic focal alterations. MRI findings, but not CT, correlated with the clinical picture.
\end{abstract}

Cerebrotendinous xanthomatosis (CTX) is a rare inherited disorder due to an enzymatic defect on the bile acid synthetic pathway. ${ }^{1}$ Sterol storage is the hallmark of the disease and results in the formation of xanthomas in the brain, lungs and tendons. ${ }^{23}$ High levels of plasma cholestanol are diagnostic. ${ }^{1}$ Clinical presentation includes juvenile cataract, enlargement of Achilles tendons and various combinations of neurological signs and symptoms. $^{2-5}$ Replacement treatment with chenodeoxycholic acid has proven clinically effective. ${ }^{67}$

We describe morphological alterations of the brain in a patient with CTX, as detected in vivo by means of noninvasive neuroradiological investigations.

Clinica Neurologica, ${ }^{\star}$ Servizio Autonomo di Neuroradiologia, $\uparrow$ Dipartimento di Scienze Neurologiche, Università degli Studi di Roma "La Sapienza", Rome and SIstituto di Scienze Neurologiche and Centro per lo Studio delle Encefalo-MieloNeuropatie Genetiche dell'Università di Siena, Siena, Italy. M Fiorelli^

V Di Piero*
L Bozzao ${ }^{\star}$

$S$ Bastianello $†$

A Federico§

Correspondence to:

Dr Bastianello, Servizio

Autonomo di

Neuroradiologia,

Departimento di Scienze

Neurologiche, Universit degli Studi di Roma "La Sapienza", $V$ le

dell'universita 30, Rome, Italy.

Received 28 February 1989 Accepted 1 September 1989 extensor plantar responses. Also present were masking of the face, infrequent blinking and cogwheel phenomenon on passive movements of wrists and elbows. Speech was slow and slurred but without scanning or explosive features. Coordination and eye movements were unimpaired. Clinical testing of sensory function did not yield reliable results because the patient could not cooperate. On general examination nothing abnormal was noted apart from a marked enlargement of the Achilles tendons.

Routine laboratory examination revealed no alteration; in particular, liver function as evaluated by serum chemistry, was normal. Blood cholesterol was $4 \cdot 7 \mathrm{mmol} / 1$ and triglycerides $1.4 \mathrm{mmol} / 1$. Biochemical screening for Wilson's disease was unrevealing. Plasma cholestanol level was increased $(0.043$ $\mathrm{mmol} / \mathrm{l}$; normal value ${ }^{1}$ : $\left.<0.026 \mathrm{mmol} / \mathrm{l}\right)$. Ultrasound examination of the liver and biliary tree, ECG, echocardiography, chest radiographs and CSF were normal. EEG showed slow waves in the temporal regions. EMG and nerve conduction velocity suggested a mild demyelinating sensory-motor neuropathy. Latencies of brainstem acoustic evoked potentials were bilaterally increased but arm somatosensory evoked potentials were normal.

CT examination showed that the cerebellar white matter was diffusely hypodense with central areas of mild relative hyperdensity (fig). No modification was observed after contrast injection. Cerebellar folia were atrophic as well as frontotemporal cortex. The rest of the examination was unrevealing.

MRI was performed using a Philips Gyroscan imager, with a superconducting magnet operating at 1.5 tesla. A T1-weighted sequence showed two hypointense symmetrical lesions in cerebellar white matter (fig) and unremarkable supratentorial images. Intermediate and T2weighted images revealed two ovoid cerebellar lesions with well-defined edges, in which a hyperintense core and a marked hypointense rim could be detected (fig). Focal hyperintensities were present in the mesencephalon and in the globus pallidus bilaterally. Some increased signal was also in evidence in the peritrigonal white matter.

At the end of the diagnostic workup the patient was put on oral chenodeoxycholic acid $250 \mathrm{mg}$ three times a day.

\section{Discussion}

The demonstration that therapy with chenodeoxycholic acid can halt the progression of CTX and reverse some of the neurological 
Figure Axial CT and $M R$ images of a CTX patient. See text for interpretation. a) Noncontrast CT shows diffuse hypodensity of the cerebellar white matter surrounding two symmetrical paramedian areas of relatively increased attenuation. MRI through the same level shows $b$ ) (T1-SE 480/20) low signal intensity in the cerebellar white matter and c) (T2-SE 1800 100) a bilateral cerebellar lesion with an eccentric portion of high signal intensity surrounded by a marked hypointense rim, which is more prominent ventrally. Supratentorial cuts show d) (T2-SE 1800/100) bilateral slitlike areas of increased signal in the region of the globus pallidus and $e$ ) (proton density-SE $1800 / 50)$ increased signal in the cerebral peduncles and the peritrigonal white matter. T1-weighted images and CT through d) and e) were unrevealing.
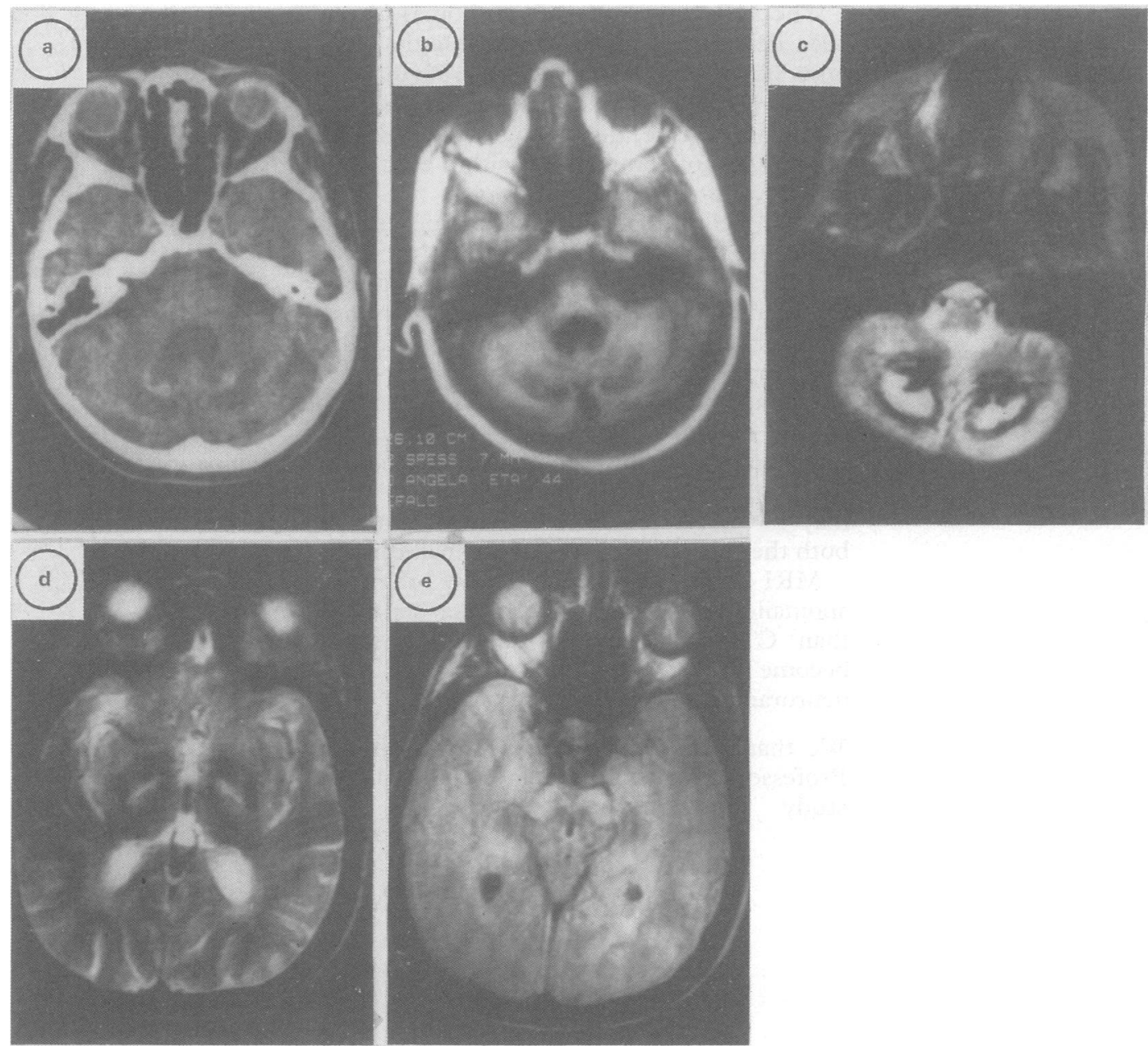

signs ${ }^{67}$ has greatly raised the interest towards this rare disease.

In CTX, sterol storage causes the formation of brain xanthomas. These lesions have a different shape and size but a constant location. $^{234}$ Pathological examination shows that the involvement of the cerebellum is prominent and results from extensive granulomatous lesions that replace the cerebellar white matter. ${ }^{234}$ Smaller lesions in other areas of the CNS always coexist but are often visible only after appropriate staining. ${ }^{4}$ Demyelination and fat-laden mononuclear cells are found in lesions of any size, while necrosis, gliosis and cystic spaces are typical of large cerebellar lesions.

Necropsy examinations in CTX demonstrate dissemination of focal lesions in the central nervous system, ${ }^{2-4}$ but neuroradiological reports have so far failed to disclose the whole pathological spectrum of the disease. Out of nine patients, Berginer et al, found on CT diffuse hypodensity of the cerebral white matter in seven; in five cases similar hypodensity also involved the cerebellum. Waterreus $e t a p^{p}$ found cerebellar white matter hypodensity in 8 of 16 cases and no other anomalies. As to focal lesions, in one of the cases of Berginer et al $^{\beta}$ a discrete cerebellar hypodensity could be interpreted as a xanthoma because of the presence of a slight mass effect; interestingly the lesion disappeared after treatment. ${ }^{6}$ Canelas $e t$ al described two sisters in which CT disclosed two hyperdense cerebellar lesions in one and a small parietal calcifications in the other case. Cromwell and Swanson ${ }^{10}$ observed a case in which MRI detected a diffuse hyperintense signal in $\mathrm{T} 2$-weighted images through the supratentorial white matter, consistent with demyelination. No focal lesions were seen, probably due to the use of a low-strength resistive magnet $(0 \cdot 15$ Tesla). In another case report, ${ }^{7}$ a brain MRI is cited, showing mild atrophy but no demyelination. So far, studies comparing radiological and anatomical findings are lacking. This is possibly due to the rarity of CTX and to the difficulty of obtaining pathology in vivo, as neither cerebral biopsy nor surgery have a role in the management of this disease.

In our patient, both MRI and CT showed a cerebellar involvement but MRI detected additional extracerebellar lesions. Pallidal and mesencephalic abnormalities as well as the core of the cerebellar lesions had specific features (hypointensity in $\mathrm{T} 1-$ and hyperintensity in T2-weighted images), suggesting necrosis and/ or demyelination. On the other hand, the appearance of the rim of the cerebellar lesions (hypointensity in T1 and T2) suggested either a relative lack of proton density which might be due to calcification or a paramagnetic effect as is seen with hemosiderin. While calcification was excluded by $C T$, the presence of hemosiderin remains a possibility. Necropsy studies $^{2-4}$ have shown both xanthomas and demyelination in these sites. 
The MR appearance of xanthomas has not been determined but it is probable that, as in other heterogenous brain lesions rich in sterols such as epidermoid cysts, the signal would vary from case to case, depending on the different proportions of components within each lesion (sterols, proteins, calcium). ${ }^{11}$

From a clinical point of view our observation confirms that in CTX a severe cerebellar degeneration can be asymptomatic, as already reported by Waterreus et al. ${ }^{5}$ Very early onset, probably before birth, and subsequent functional adaptation could explain this apparent paradox. On the other hand, in our patient, the spastic tetraparesis could be due to involvement of the cerebral peduncles and parkinsonism, never reported so far in CTX, may have resulted from the involvement of both the substantia nigra and the pallidum. ${ }^{12}$

MRI detects clinically relevant brain focal anomalies in CTX with a higher sensitivity than CT. ${ }^{10}$ We suggest that MRI should become the investigation of choice in the neuroradiological assessment of CTX.

We thank Dr G Salen, Dr U Salvolini and Professor C Fieschi for their support with this study.
1 Salen G, Shefer S, Berginer VM. Familial diseases with storage of sterols other than cholesterol: cerebrotendinous xanthomatosis and sitosterolemia with xanthomatosis. In: Stanbury JB, Wyngaarden JB, Fredrickson DS, Goldstein JL, Brown MS, eds. The metabolic basis of Goldstein JL, Brown MS, eds. The metabolic basis of 1983:713-30.

van Bogaert L, Scherer HJ, Epstein E. Une forme cérébrale de la cholestérinose généralisée. Paris: Masson et Cie, 1937.

3 van Bogaert L, Scherer HJ, Froelich A, Epstein E. Une deuxième observation de cholestérinose tendineuse symétrique avec symptômes cérébraux. Ann Med 1937;42:69-101.

4 Schimschock JR, Alvord EC Jr, Swanson PD. Cerebrotendinous xanthomatosis. Clinical and pathological studies. Arch Neurol 1968;18:688-98.

5 Waterreus RJ, Koopman BJ, Wolthers BG, Oosterhuis HJGH. Cerebrotendinous xanthomatosis (CTX): A clinical survey of the patient population in The Netherlands. Clin Neurol Neurosurg 1987;89(2):169-75.

6 Berginer VM, Salen G, Shefer S. Long-term treatment of Berginer VM, Salen G, Shefer S. Long-term treatment of
cerebrotendinous xanthomatosis with chenodeoxycholic cerebrotendinous xanthomatosis with

7 Pedley TA, Emerson RG, Warner CL, Rowland LP, Salen G. Treatment of cerebrotendinous xanthomatosis with chenodeoxycholic acid. Ann Neurol 1985;18:517-8.

8 Berginer VM, Berginer J, Salen G, Shefer S, Zimmermann RD. Computed Tomography in cerebrotendinous xanthomatosis. Neurology (Ny) 1981;31:1463-5.

9 Canelas HM, Quintão ECR, Scaff M, Vasconcelos KS Brotto MWI. Cerebrotendinous xanthomatosis: clinical and laboratory studies of 2 cases. Acta Neurol Scand 1983;67:305-11.

10 Swanson PD, Cromwell LD. Magnetic resonance imaging in cerebrotendinous xanthomatosis. Neurology 1986;36: $124-6$.

11 Vion-Dury J, Vincentelli F, Jiddane $M$ et al. MR imaging of epidermoid cysts. Neuroradiology 1987;29:333-8.

12 Jellinger $K$. Exogenous lesions of the pallidum. In: Vinken PJ, Bruyn GW, Klawans HL, eds. Handbook of clinical neurology (Vol 49). Amsterdam: Elsevier, 1986:465-92. 